\title{
PLANTAS MEDICINALES EN UNA ALDEA DEL ESTADO DE TABASCO, MÉXICO
}

\author{
MEDICINAL PLANTS IN A SMALL VILLAGE IN THE STATE OF TABASCO, MÉXICO
}

\author{
Regino Gómez Álvarez
}

El Colegio de la Frontera Sur (ECOSUR).Carretera a Reforma Km 15.5 s/n, Ranchería Guineo Segunda sección, Municipio Centro, Villahermosa, Tabasco, México. Teléfono. Fax 9933136110 extensión 3403.

Autor para correspondencia (regomez@ecosur.mx)

\section{RESUMEN}

El uso de plantas medicinales es resultado de la experiencia e íntimo contacto con la naturaleza que el hombre ha acumulado por generaciones, así como de la convivencia entre las culturas de diferentes pueblos. Este saber ha permitido que sobrevivan comunidades que habitan en lugares apartados, donde hay carencias de servicios médicos y donde las parteras, "yerberos" y curanderos son los principales responsables de la salud de los habitantes. En este estudio se hicieron colectas de plantas y se entrevistó a 20 familias, dos parteras, un Comisario Ejidal y a una responsable del Grupo de Mujeres que producen preparados naturales. La información se procesó y documentó, con el apoyo de especialistas en etnobotánica, visitas a herbarios y consultas en universidades y centros de investigación. Los resultados muestran 112 plantas medicinales que se emplean para curar diversos malestares, que se agrupan en $\mathbf{5 7}$ familias botánicas en las que prevalecen las herbáceas y arbóreas. En su mayoría se usan las hojas en cocimientos para elaborar los medicamentos. El conocimiento del uso de las plantas proviene principalmente de los abuelos, padres y vecinos, y la mayoría de las plantas crecen en los huertos familiares de la comunidad.

Palabras claves: Etnobotánica, plantas medicinales, remedios naturales

\section{SUMMARY}

The use of medicinal plants is the result of the experience and intimate contact of human beings with nature and the coexistence among different cultures. This wisdom has permitted the survival of communities living in remote areas, where medical services are scarce, and midwives, "herbalists" and folk healers are responsible for the people health. In this study, plants were collected and 20 families, two midwives, one Ejido Sheriff, and one representative of a group of women that produce natural preparations, were interviewed. The information was processed and documented with the support of specialists in ethnobotany, visits to plant collections and enquiries at universities and research centers. Results show that $\mathbf{1 1 2}$ medicinal plants are used for alleviating medical problems. These plants are grouped in 57 botanical families, most of them herbaceous and arborous. Leaf infusions are the most common way for making medicines. The knowledge of these plants and their uses come mainly from grandparents, parents and neighbors, and most of the plants are grown in the village.

Index words: Ethnobotany, medicinal plants, natural medicines

\section{INTRODUCCIÓN}

En la actualidad existe un interés creciente en la medicina alternativa para la cura de un numerosos padecimientos y enfermedades que afectan a los seres humanos, por lo que las investigaciones que tengan como objetivo el cultivo, estudio y procesamiento de plantas medicinales con fines terapéuticos se consideran estratégicas e importantes (Soto et al., 2002). En las comunidades carentes de servicios médicos, las parteras, los “yerberos" y los curanderos son los responsables de la salud de los pobladores. Incluso en lugares donde hay servicios médicos, los recursos de la medicina tradicional son utilizados por las personas enfermas. En las áreas marginadas existen grupos indígenas con grandes conocimientos de la herbolaria medicinal y del ambiente donde se desarrollan estas plantas. Tales conocimientos acumulados por muchas generaciones constituyen la base de la biodiversidad cultural global y del uso sustentable de estos recursos (Caballero y Cortés, 2001; Leonti et al., 2003; Canales et al., 2006). Para estudiar las plantas medicinales es indispensable la exploración etnobotánica, que incluye la colección, propagación y conservación de las especies (Hernández, 1985; Ocampo, 1994).

México es uno de los países de América con mayor tradición ancestral y riqueza en el uso de la herbolaria medicinal, donde se registran poco más de 3000 especies que se emplean en remedios naturales. No obstante, son pocas las investigaciones en el uso y manejo de las plantas medicinales, y por tanto, hay escasa información etnobotánica en este tema (Linares et al., 1999; Casas et al., 2001; Dávila et al., 2002; Hernández et al., 2005). La información que se pueda recopilar en las diversas regiones del país tendría relevancia etnobotánica y serviría para definir estrategias que mejoren el aprovechamiento y manejo de los recursos de la flora medicinal.

Si bien se han logrado grandes avances de la medicina 
científica, la cobertura de los programas de salud en México aún es insuficiente. En el Estado de Tabasco, uno de los más pequeños del país en extensión territorial, existen 1585 comunidades rurales dispersas con menos de 500 habitantes (INEGI, 2010). La dispersión de las comunidades y la falta de tecnificación de la agricultura son dos características de Tabasco, lo que representa serios obstáculos para la prestación de los servicios del sector salud, y por ello es necesario valorar la medicina tradicional como coadyuvante para resolver este problema en la entidad.

En las comunidades rurales las plantas medicinales se usan para la cura de diferentes malestares, uso que está asociado con la presencia de curanderos, muchos de los cuales poseen un profundo conocimiento de la herbolaria (Casas et al., 2001; Canales et al., 2006; Monroy y Castillo, 2007). En varios municipios de Tabasco se ha iniciado un proceso de recuperación del germoplasma medicinal y de sistematización del conocimiento mediante la publicación de manuales y artículos científicos donde se documenta la experiencia acumulada en el uso de la medicina naturista (Magaña et al., 2010).

En Nacajuca, Tabasco, Magaña et al. (2010) clasificaron varias plantas medicinales y detallaron cómo se conservan y propagan, pero sin cubrir aspectos relevantes tales como: dónde se encuentran las plantas más usadas por los pobladores de las comunidades, cómo se conserva y transmite el conocimiento de la herbolaria medicinal, y qué parte de las plantas son las más utilizadas, entre otros.

El presente estudio se coordinó con organizaciones de productores del Municipio Centro de Tabasco, que poseen microempresas para la producción artesanal de medicamentos naturales con base en la herbolaria de la región. En el desarrollo del proyecto participaron de forma activa los productores para determinar las plantas medicinales disponibles, su clasificación taxonómica, los usos que los pobladores les dan, las formulaciones para la preparación de medicamentos naturales, así como diversos aspectos del manejo de la herbolaria medicinal.

\section{MATERIALES Y MÉTODOS}

Descripción del área de estudio. La Ranchería Corregidora Ortiz $1^{\text {ra }}$ Sección está a $32 \mathrm{~km}$ del Municipio de Centro, Tabasco, sobre la carretera Villahermosa-Reforma. Se localiza en las coordenadas $93^{\circ} 06^{\prime} 31^{\prime \prime} \mathrm{LO}$ y $17^{\circ} 54^{\prime} 32^{\prime \prime}$ $\mathrm{LN}$, a una altitud de $30 \mathrm{~m}$. Al norte colinda con pequeñas propiedades de la Ranchería Cumuapa, al este con la Ranchería Río Tinto 1ra Sección, al sur con la Ranchería Miguel Hidalgo y al oeste con la Ranchería Corregidora Ortíz 2da Sección (AGEB, Análisis Geo Estadísticos Básicos, INEGI, 2010).
El suelo predominante de la Ranchería es un Gleysol éutrico, de textura arcillosa, con problemas de exceso de humedad por drenaje deficiente. Este suelo posee un horizonte superficial bien estructurado, de color oscuro, con una saturación de bases de 50 \% (FAO, 1999; PalmaLópez y Cisneros-Domínguez, 2000). El clima es cálido húmedo con abundantes lluvias en verano; la temperatura anual oscila entre $33.6^{\circ} \mathrm{C}$ en mayo y $21.7^{\circ} \mathrm{C}$ en diciembreenero. La precipitación anual promedio es de $2237 \mathrm{~mm}, \mathrm{y}$ septiembre es el mes más lluvioso. Los vientos dominantes son en dirección noroeste, acompañado de lluvias continuas ("nortes") de octubre a marzo (Palma-López y CisnerosDomínguez, 2000).

Se entrevistaron 20 familias que representan cerca de $20 \%$ de la población económicamente activa. Además se entrevistó a dos parteras, un Comisario Ejidal y a una Responsable del grupo de mujeres que producen preparados naturales. Las entrevistas se basaron en un cuestionario en el que se registraron los siguientes datos: los nombres del encuestador y del encuestado; información general del agro-ecosistema; tipo de propiedad, especies de plantas colectadas y épocas de cosecha; información sobre el sistema de producción; procedencia del conocimiento en plantas medicinales; frecuencia de uso de las plantas medicinales y tipo biológico de las mismas; cultivos cercanos a la zona donde se siembran las plantas medicinales; partes de las plantas que se usan para elaborar las recetas naturistas, cómo preparar las mismas y para qué enfermedades se recomiendan; frecuencia de uso del servicio médico estatal; $y$ conocimientos que se tienen sobre los curanderos y yerberos.

Posteriormente se hicieron colectas de las plantas mencionadas en las entrevistas (20 individuos por especie); se tomaron fotografías y las imágenes se guardaron en archivos electrónicos. Finalmente, las plantas seidentificaron por comparación visual con datos de herbarios, manuales, diccionarios botánicos y artículos científicos. En las colectas de campo se utilizaron las técnicas convencionales para estudios florísticos (Lot y Chiang, 1986). Se consultó el herbario de la División Académica de Ciencias Biológicas (DACB) de la Universidad Juárez Autónoma de Tabasco (UJAT), así como las bibliotecas de El Colegio de la Frontera Sur (ECOSUR), Instituto Tecnológico de la Zona Olmeca de Tabasco (ITZO) y de la DACB. La información obtenida en el trabajo de campo se organizó en cuadros conforme al método etnobotánico de Kavist et al. (2001) y Giménez (1994), donde se anotan las acciones realizadas y las interpretaciones de las mismas.

Al listado de plantas medicinales se agregó su clasificación taxonómica con sus nombres científicos, nombres comunes, familias, formas biológicas y el uso medicinal que los 
pobladores de la comunidad les atribuyen.

\section{RESULTADOS Y DISCUSIÓN}

En los Cuadros 1, 2 y 3 se presentan las plantas medicinales registradas a través de colectas y entrevistas, así como la descripción botánica de las mismas. Se encontraron 112 especies agrupadas en 57 familias botánicas, entre las que sobresalen: Asteraceae (10), Lamiaceae (7), Fabaceae (5), Malvaceae (5), Rutaceae (5), Bignoniaceae (4), Euphorbiaceae (4), Verbenaceae (4), Amaryllidaceae (3), Piperaceae (3) y Solanaceae (3), los cuales variaron entre tres y diez especies por familia. Le siguen, en orden decreciente, con dos especies por familia: Acanthaceae, Annonaceae, Bixaceae, Boraginaceae, Burseraceae, Crassulaceae, Curcurbitaceae, Lauraceae, Myrtaceae, Poaceae, Rosaceae, Sapotaceae y Vitaceae,. Las familias restantes están representadas con una sola especie. De acuerdo con las entrevistas, a estas especies se les atribuyen propiedades curativas para un gran número de malestares, como infecciones de la piel, del estómago y de los riñones; inflamación del vientre, oídos y encías; dolores musculares y de muelas; quemaduras; tos; nerviosismo; fiebres; cicatrización de heridasy reuma, entre otras.

En trabajos previos realizados por la UJAT (Alegría, 1994; Com. personal ${ }^{1}$ ), se han reportado 32 plantas medicinales en huertos de traspatio de la Chontalpa, que se usan en más de 25 padecimientos, sobre todo en problemas nerviosos, de gastritis, digestivos, infecciones de los riñones, gripe, asma, fiebres, dolores musculares, inflamación de garganta, $\mathrm{y}$ otros.

Los resultados de la entrevistas indican que $91 \%$ de la población de la localidad resuelven sus padecimientos con recetas de plantas medicinales, y sólo van al médico cuando la afectación es muy grave, o no la pueden controlar. Todas las personas entrevistadas obtienen las plantas en la propia comunidad, lo que evidencia la amplia biodiversidad vegetal presente en esa Ranchería, y el gran conocimiento que el campesino mexicano tiene del uso de la flora medicinal y de recetas naturistas para aliviar sus dolencias más frecuentes. El germoplasma que obtienen por compra en el mercado o por trueque es insignificante en comparación con los existentes en la propia comunidad, aunque ocasionalmente han adquirido germoplasma específico que no se siembra en la región.

Con respecto a los tipos biológicos de las especies que componen la herbolaria medicinal, $42 \%$ fueron herbáceas, seguidas por las arbóreas con $24 \%$, y por las arbustivas

\footnotetext{
${ }^{1}$ Alegría P (1994). Uso actual de las plantas medicinales en la Ranchería Tumbulushal, Centro, Tabasco, México. Tesis de Licenciatura. Universidad Juárez Autónoma de Tabasco. División Académica Ciencias Biológicas, Villahermosa, Tabasco. 72 p.
}

con $25 \%$; el restante (9\%) son trepadoras y tubérculos. Referente a la ubicación de las especies curativas, $55 \%$ de ellas se encuentran en huertos familiares, seguido de los acahuales (zona de transición entre la selva y los terrenos agrícolas donde la vegetación surge de manera espontánea) con $18 \%$, potreros $18 \%$, y en zonas agrícolas $9 \%$. Algunas especies no se encuentra en el municipio debido a que no se adaptan a esas condiciones ambientales, como es el caso de la manzanilla (Matricaria chamomilla L.), que se adquiere en tiendas o en el mercado local. Lo descrito corrobora la importancia de la relación hombre-planta medicinal. Según los entrevistados, el conocimiento para el uso de la medicina verde se originó de los abuelos (37\%), de los padres (27\%) y $36 \%$ de otras fuentes (vecinos, parientes, revistas, etc.). Es decir, la medicina tradicional no es una manifestación carente de dinamismo, sino que es un proceso muy activo, cambiante, y de trascendencia cultural, como indicaron Cervantes y Valdés (1990).

Los estudios realizados en el Estado de Tabasco y en otras entidades de México, coinciden en señalar que el lugar principal donde se encuentran las plantas medicinales utilizadas por los habitantes son los huertos familiares, lugares donde fluye la transmisión y adopción del conocimiento de la herbolaria medicinal. Según Magaña et al. (2010), las personas de más edad son las que poseen los mayores conocimientos de la medicina naturista en lo referente al uso, siembra, fertilización, riego, cosecha y conservación de las plantas; además, esas personas conocen en detalle las formas de preparar los remedios.

En el presente estudio, $64 \%$ del conocimiento provino de padres y abuelos. En Nacajuca, Tabasco, Magaña et al. (2010) reportaron 173 especies, cantidad mayor que las 112 registradas en Corregidora Ortíz $1^{\text {ra }}$ Sección, lo que se atribuye a que muchas plantas medicinales no son utilizadas por los pobladores de esta Ranchería porque desconocen sus propiedades curativas, aspecto confirmado en las encuestas realizadas. En el estudio de Magaña et al. (2010), los entrevistados también informaron que $25 \%$ de las plantas presentes de Nacajuca se compran en los mercados o se traen de otros lugares, mientras que en Corregidora Ortíz 1ra Sección sólo $9 \%$ provienen de fuera de la localidad. Los trabajos de Pérez et al. (2005); Castellanos de los Santos (2008 Com. personal²) y Sánchez (2008; Com. personal $\left.3^{3}\right)$, coinciden en afirmar que se tienen registros

\footnotetext{
${ }^{2}$ Castellanos de los Santos G (2008) Uso de las plantas medicinales en la Ranchería Centro Tular 1ra Sección del Municipio de Comalcalco, Tabasco. Tesis de Licenciatura. Universidad Juárez Autónoma de Tabasco. División Académica Ciencias Biológicas. Villahermosa, Tabasco. 47 p.
}

${ }^{3}$ Sánchez R F R (2008) Uso y manejo de las plantas medicinales de cinco comunidades aledañas a la Villa de Tamulté de las Sabanas, Centro, Tabasco, México, Tesis de Licenciatura, Universidad Juárez Autónoma de Tabasco, División Académica Ciencias Biológicas. Villahermosa, Tabasco. $51 \mathrm{p}$. 
Cuadro 1. Nombres comunes, científicos, familias y formas biológicas de plantas medicinales encontradas en la Ranchería Corregidora Ortiz Primera Sección, Tabasco, México.

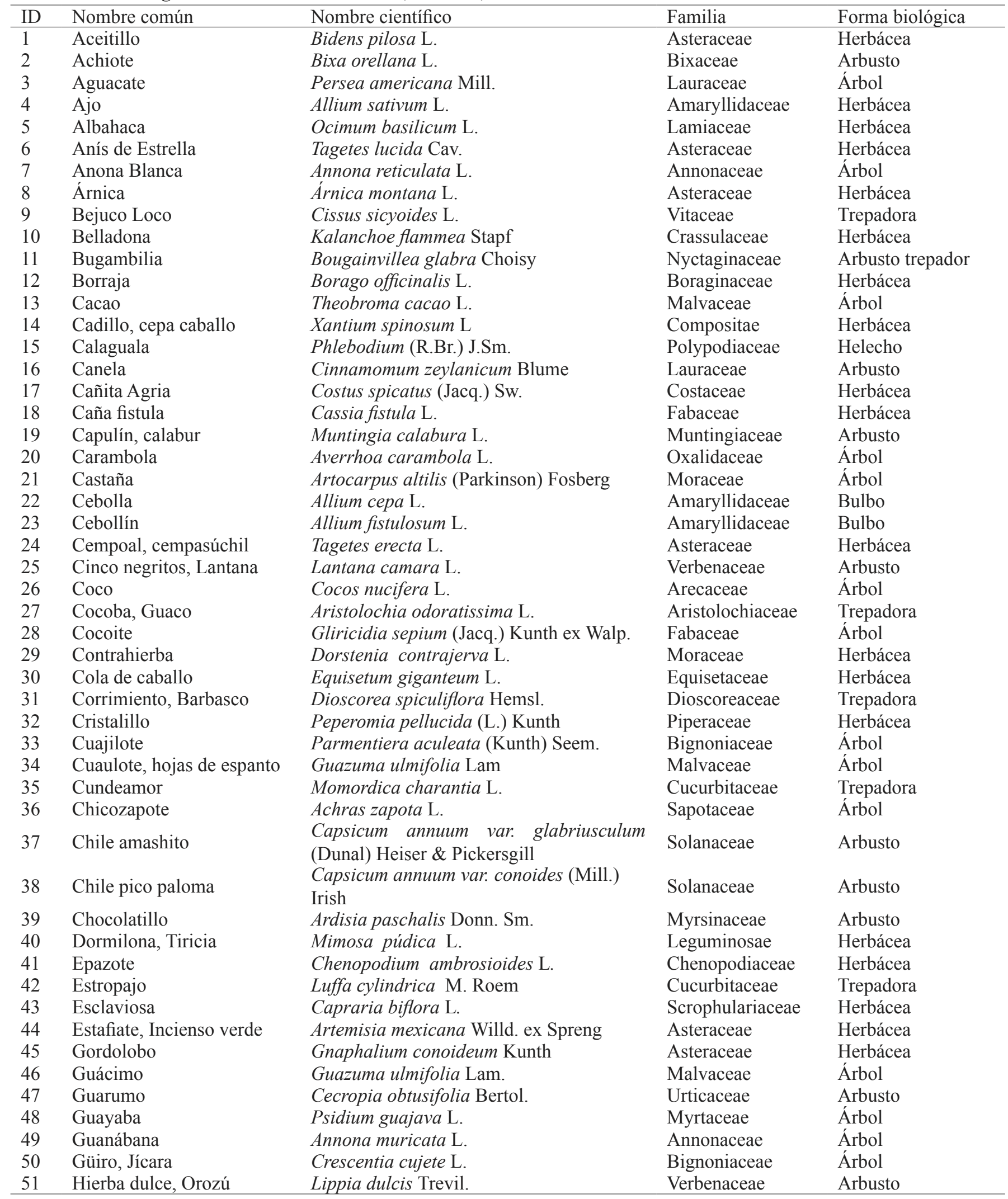


Cuadro 2. Nombres comunes, científicos, familias y formas biológicas de plantas medicinales encontradas en la Ranchería Corregidora Ortiz Primera Sección, Tabasco, México.

\begin{tabular}{|c|c|c|c|c|}
\hline ID & Nombre común & Nombre científico & Familia & Forma biológica \\
\hline 52 & Hierba Martín & Hyptis verticillata Jacq. & Lamiaceae & Herbácea \\
\hline 53 & Higuerilla, Higuera & Ricinus communis L. & Euphorbiaceae & Árbol \\
\hline 54 & Higuerilla negra & Micrandra spruceana (Baill.) R.E. Schultes & Euphorbiaceae & Árbol \\
\hline 55 & Hoja fresca, canzera & Tournefortia glabra L. & Boraginaceae & Arbusto \\
\hline 56 & Hormiguera & Senna occidentalis (L.) Link & Fabaceae & Herbácea \\
\hline 57 & Granadilla gigante, Badea & Passiflora quadrangularis L. & Passifloraceae & Trepadora \\
\hline 58 & Jovillo; Ron ron & Astronium graveolens Jacq. & Anacardiaceae & Árbol \\
\hline 59 & Justicia, Trébol & Justicia comata (L.) Lam. & Acanthaceae & Herbácea \\
\hline 60 & Lima, Lima de Chiche & Citrus limetta Risso & Rutaceae & Árbol \\
\hline 61 & Limón Agrio & Citrus limon (L.) Burm. f. & Rutaceae & Árbol \\
\hline 62 & Limón Dulce & Citrus limettioides Tanaka & Rutaceae & Árbol \\
\hline 63 & Llantén & Plantago major $\mathrm{L}$. & Plantaginaceae & Herbácea \\
\hline 64 & Maculí, Palo de rosa & Tabebuia rosea (Bertol.) A. DC. & Bignoniaceae & Árbol \\
\hline 65 & Guayacán rosado & Tabebuia rosea Sw. & Bignoniaceae & Árbol \\
\hline 66 & Malva Silvestre & Malva sylvestris $\mathrm{L}$. & Malvaceae & Herbácea \\
\hline 67 & Malvarisco & Corchorus siliquosus L. & Malvaceae & Arbusto \\
\hline 68 & Manzanilla & Matricaria chamomilla $\mathrm{L}$. & Asteraceae & Herbácea \\
\hline 69 & Mastuerzo & Cleome serrata Jacq. & Cleomaceae & Herbácea \\
\hline 70 & Matalí, Cucaracha & Tradescantia zebrina D.R. Hunt & Commelinaceae & Herbácea \\
\hline 71 & Mayorca, Patitos & Pedilanthus tithymaloides (L.) Poit. & Euphorbiaceae & Arbusto \\
\hline 72 & Maíz & Zea mays $\mathrm{L}$. & Poaceae & Herbácea \\
\hline 73 & $\begin{array}{l}\text { Margarita mayor, } \\
\text { Margarita }\end{array}$ & Chrysanthemum leaucanthemum L. Bernh & Asteraceae & Arbusto \\
\hline 74 & Mazorquilla & Blechum brownei Juss. & Acanthaceae & Arbustiva \\
\hline 75 & Momo de Ombligo & Piper umbellatum $\mathrm{L}$. & Piperaceae & Arbusto \\
\hline 76 & $\begin{array}{l}\text { Momo, Hoja santa, } \\
\text { Caisimón de anís }\end{array}$ & Piper auritum Kunth & Piperaceae & Arbusto \\
\hline 77 & Rosa mosqueta, Isabelita & Rosa $\mathrm{L}$. & Rosaceae & Arbusto \\
\hline 78 & Nance & Byrsonima crassifolia (L.) Kunth & Malpighiaceae & Árbol \\
\hline 79 & Naranja Agria & Citrus aurantium L. & Rutaceae & Árbol \\
\hline 80 & Naranja Grey, Pomela & Citrus grandis (L.) Osbeck & Rutaceae & Árbol \\
\hline 81 & Nopal & Opuntia decumbens Salm-Dyck & Cactaceae & Arbusto \\
\hline 82 & Oreganón & Lippia graveolens Kunth & Verbenaceae & Herbácea \\
\hline 83 & Palo Mulato & Bursera simaruba (L.) Sarg. & Burseraceae & Árbol \\
\hline 84 & Papaloquelite, Mata dolor & Porophyllum ruderale (Jacq.) Cass. & Asteraceae & Herbácea \\
\hline 85 & Papaya & Carica papaya $\mathrm{L}$. & Caricaceae & Arbusto \\
\hline 86 & Perejil & Eryngium foetidum $\mathrm{L}$. & Apiaceae & Herbácea \\
\hline 87 & Pericón, San Nicolás & Kalanchoe pinnata (Lam.) Pers. & Crassulaceae & Herbácea \\
\hline 88 & Pimienta, Pimentón & Pimenta dioica L. Merr. & Myrtaceae & Herbácea \\
\hline 89 & Plátano & Musa paradisiaca L. & Musaceae & Arbusto \\
\hline 90 & Poleo & Mentha pulegium L. & Lamiaceae & Herbácea \\
\hline 91 & Riñonina, Riñoncito & Ipomea pes-caprae (L.) R. Br. & Convolvulaceae & Planta rastrera \\
\hline 92 & Ruda & Ruta graveolens $\mathrm{L}$. & Rutaceae & Herbácea \\
\hline 93 & Rosa, Rosa Concha & Hibiscus rosa-chinensis L. & Rosaceae & Arbusto \\
\hline 94 & Sábila & Aloe vera $\mathrm{L}$. ex Webb & Xanthorrhoeaceae & Herbácea \\
\hline 95 & Sánalo todo, bejuco loco & Cissus sicyoides L. & Vitaceae & Trepadora \\
\hline 96 & Salvia real & Salvia officinalis L. & Lamiaceae & Herbácea \\
\hline 97 & Salvia cruz, rabo de gato & Salvia leucantha Cav. & Lamiaceae & Arbusto \\
\hline 98 & Sapillo, hierba de sapo & Epaltes mexicana Less. & Asteraceae & Arbusto \\
\hline 99 & Sasafrás & Bursera graveolens (Kunth) Triana et Planch & Burseraceae & Arbusto \\
\hline 100 & Saúco & Sambucus mexicana C. Presl ex DC. & Adoxaceae & Árbol \\
\hline 101 & Tabaco & Nicotiana tabacum L. & Solanaceae & Arbusto \\
\hline
\end{tabular}


Cuadro 3. Nombres comunes, científicos, familias y formas biológicas de plantas medicinales encontradas en la Ranchería Corregidora Ortiz Primera Sección, Tabasco, México.

\begin{tabular}{lllll}
\hline ID & Nombre común & Nombre científico & Familia & Forma biológica \\
\hline 102 & Tamarindo & Tamarindus indica L. & Fabaceae & Árbol \\
103 & Tinto, palo de tinte & Haematoxylum campechianum L. & Fabaceae & Árbol \\
104 & Toronjil, Hierba buena & Mentha citrata Ehrh. & Lamiaceae & Herbácea \\
105 & Toronjil de Menta & Melissa officinalis L. & Lamiaceae & Herbácea \\
106 & Tulipán & Hibiscus rosa sinensis L. & Malvaceae & Arbusto \\
107 & Verbena & Stachytarpheta jamaicensis L.) Vahl & Verbenaceae & Herbácea \\
108 & Verdolaga & Portulaca oleracea L. & Portulacaceae & Herbácea \\
109 & Vicaria & Catharanthus roseus (L.) G. Don & Apocynaceae & Arbusto \\
110 & Zacate Limón & Cymbopogon citratus (DC.) Stapf & Poaceae & Herbáceo \\
111 & Zapote, Chicozapote & Pouteria sapota (Jacq.) H.E. Moore \& Stearn & Sapotaceae & Árbol \\
112 & Zorrillo & Petiveria alliacea L. & Phytolaccaceae & Herbácea \\
\hline
\end{tabular}

de entre 54 a 173 plantas utilizadas por los productores de diferentes zonas del Estado de Tabasco para preparar medicamentos naturales; estasdiferencias se atribuyen a la poca información científica de la flora medicinal de la región que tienen los productores entrevistados. Además diferentes autores (Hernández, 2006, Com. personal'; Ulin, 2006, Com. personal ${ }^{5}$ ); Castellanos de los Santos, 2008; Sánchez, 2008; Magaña et al., 2010), consignan que las plantas herbáceas son las más utilizadas para preparar los medicamentos naturales, y son las que prevalecen en los huertos familiares.

También hay coincidencia en que las partes u órganos más utilizados de las plantas herbáceas son las hojas. Muchas de estas plantas tienen propiedades como plaguicidas naturales, cuyos extractos se aplican al follaje de los cultivos (Gómez y Castañeda, 2000; Trujillo y García, 2001; Soto et al., 2002). El efecto benéfico de estas aplicaciones se debe a que estas plantas poseen metabolitos secundarios con propiedades insecticidas. Entre los extractos usados están los de higuerilla (Ricinus communis L), tabaco (Nicotiana tabacum L.), trompetera (Brugmansia candida Pers.), cola de caballo (Equisetum giganteum L.) y hoja santa (Piper auritum H.B.K), las cuales funcionan como controladores de gusanos defoliadores (Gómez y Castañeda, 2000; Trujillo y García, 2001).

\section{CONCLUSIONES}

En la Ranchería Corregidora Ortiz 1ra Sección se encontraron 112 especies que se utilizan para tratar 42 malestares. Estas especies se agrupan en 57 familias

\footnotetext{
${ }^{4}$ Hernández G M I (2006) Contribución para el uso y manejo de las plantas medicinales de la Villa Tepetitán Macuspana, Tabasco. Tesis de Licenciatura, Universidad Juárez Autónoma de Tabasco, División Académica Ciencias Biológicas, Villahermosa, Tabasco. 64 p.

${ }^{5}$ Ulin H L Y (2006) Uso y manejo actual de las plantas medicinales en el poblado Iquinuapa, Jalpa de Méndez, Tabasco. Tesis de Licenciatura. Universidad Juárez Autónoma de Tabasco, División Académica Ciencias Biológicas. Villahermosa, Tabasco. 82 p.
}

botánicas, de las cuales las más representativas, con tres a diez especies por familia, fueron: Asteraceae (10), Lamiaceae (7), Fabaceae (5), Malvaceae (5), Rutaceae (5), Bignoniaceae (4), Euphorbiaceae (4), Verbenaceae (4), Amarillidaceae (3), Piperaceae (3) y Solanaceae (3). Por tipo biológico $42 \%$ son herbáceas, $24 \%$ arbustivas, $25 \%$ arbóreas, $7 \%$ trepadoras y $2 \%$ de tubérculos.

El conocimiento ancestral proviene de los abuelos con 36 $\%$, seguido de los padres con $27 \%$, de los hijos y vecinos $28 \%$, y el resto de otras fuentes. La mayoría de las plantas crecen en la propia comunidad, y muchas de ellas se utilizan para elaborar biopesticidas naturales.

\section{AGRADECIMIENTOS}

A la Fundación Produce Tabasco A.C., la Secretaría de Desarrollo Agropecuario Forestal y Pesca (SEDAFOP), y la Dirección de Desarrollo Agropecuario del Municipio Centro, Tabasco, por el financiamiento que permitió realizar esta investigación. A los productores de plantas medicinales de Corregidora Ortíz 1ra Sección y Plátano y Cacao 1ra Sección del Municipio Centro, Tabasco, por las facilidades brindadas para la realización de esta investigación.

\section{BIBLIOGRAFÍA}

Caballero J, L Cortés (2001) Percepción uso y manejo tradicional de los recursos vegetales en México. In: Plantas Cultura y Sociedad. B Rendón, S Rebollar, J Caballero, M A Martínez (eds). Universidad Autónoma Metropolitana-SEMARNAP, México D. F. pp:79-100.

Canales M, T Hernández, J Caballero, A Romo de Vivar, A Durán, $R$ Lira (2006) Análisis cuantitativo del conocimiento tradicional de las plantas medicinales en San Rafael, Coxcatlán, Valle de Tehuacán-Cuicatlán, Puebla, México. Acta Bot. Mex. 75:21-43.

Casas A, A Valiente-Banuet, J L Viveros, P Dávila, R Lira, J Caballero, L Cortés, I Rodríguez (2001) Plant resources of the TehuacánValley, México. Econ. Bot. 55:129-166.

Cervantes L, J Valdés (1990) Plantas medicinales del distrito Ocotlán, Oaxaca. Anal. Inst. Biol. UNAM S. Bot. 60:85-103.

Dávila P, M C Arizmendi, A Valiente-Banuet, A Casas, J L Villaseñor, 
S R Lira (2002) Biological diversity in the Tehuacán-Cuicatlán Valley. Biodiv. Conserv. 11:421-442.

Food \& Agriculture Organization, FAO (1999) Base Referencial Mundial del Recurso Suelo. Informe sobre los Recursos Mundiales de Suelos $\mathrm{N}^{\circ}$ 84. Roma, Italia. $102 \mathrm{p}$.

Giménez G (1994) La teoría y el análisis de la cultura. Problemas teóricos y metodológicos. In: Metodología y Cultura. Comisión Nacional para la Cultura y las Artes, México. pp:36-66.

Gómez R, R Castañeda (2000) Tecnologías de Producción Orgánicas en las Condiciones del Trópico. ECOSUR-ISPROTAB. Edición del Gobierno del Estado de Tabasco. 91 p.

Hernández X E (1985) Exploración etnobotánica y su metodología. In: Xolocotzia, Geografía Agrícola, Tomo I. Universidad Autónoma de Chapingo, México. pp:163-188.

Hernández T, M Canales, J Caballero, A Durán, R Liras (2005) Análisis cuantitativo del conocimiento tradicional sobre plantas medicinales para el tratamiento de enfermedades gastrointestinales en Zapotitlán de las Salinas, Puebla, México. Interciencia 30:17-27.

Instituto Nacional de Estadística y Geografía, INEGI (2010) Síntesis de Información Geográfica del Estado de Tabasco, 1:20 000.

Kavist L P, I González, A Llapasca (2001) Estudio de Plantas Medicinales en la Amazonia Peruana: Evaluación de Ocho Métodos Etnobotánicos. Consejo Nacional de la Flora de México A. C., México. $142 \mathrm{p}$.

Leonti M, O Sticher, M Heinrich (2003) Antiquity of medicinal plant usage in two Macro-Mayan ethnic groups (México). J. Ethnopharmacol. 88:119-124.
Linares D, R Bye, B Flores (1999) Plantas Medicinales de México. Usos, Remedios y Tradiciones. Instituto de Biología, UNAM, México. $155 \mathrm{p}$.

Lot E, F Chiang (1986) Comps. Manual de Herbario. Consejo Nacional de la Flora de México A. C., México. pp:1-42.

Magaña A M A, L Gama, R Mariaca (2010) El uso de las plantas medicinales en las comunidades Maya-Chontales de Nacajuca, Tabasco, México. Polibotánica 29:213-262.

Monroy C, P Castillo (2007) Plantas Medicinales Utilizadas en el Estado de Morelos. 2da ed. Universidad Autónoma de Morelos, CONABIO. 405 p.

Ocampo R (1994) Estudio etnobotánico de las plantas empleadas por los indígenas en Talamanca, Costa Rica. Rev. For. Centroam. 3:16-21.

Palma-López D J, J Cisneros-Domínguez (2000) Plan de Uso Sustentable de los Suelos de Tabasco. Vol. 1, 2da ed. Fundación Produce Tabasco A.C., Villahermosa, Tabasco, México. 115 p.

Pérez L A, M Souza, A M Hanan, F Chiang, P Tenorio (2005) Vegetación terrestre. In: Biodiversidad del Estado de Tabasco. J Bueno, F Álvarez, S Santiago (eds). Instituto de Biología. UNAM, CONABIO, México. pp:65-110.

Soto R, G Vega, A L Tamajón (2002) Instructivo técnico del cultivo de Cymbopogon citratus (D. C.) Stapf (caña santa). Rev. Cubana Plantas Med. 7:89-95.

Trujillo R J, L E García (2001) Conocimiento indígena del efecto de plantas medicinales locales sobre las plagas agrícolas en los altos de Chiapas, México. Agrociencia 35:685-692. 